\title{
XXXIV.
}

Aus der medicinischen Klinik zu Marburg a. L.

(Director: Prof. Dr. Brauer.)

\section{Nachweis, Entstehung und Bedeutung der temporären Lungenblähung.}

\author{
Von \\ Dr. Oskar Bruns, Priv.-Doc. und Oberarzt der Klinik. \\ (Hierzu Tafel XII.)
}

Die Totalcapacität der Iungen, wie sie sich aus der spirometrischen Bestimmung der Residuallu(t und Vitalcapacität's) ergiebt, war schon im 18. Jahrhundert ziemlich richtig bekannt (Haller, St. Hales, Davy). Die Schwankungen des Lungenvolumens lernte man jedoch erst durch die Arbeiten von Panum und Isovén kennen (1868 und 1872). Diese Forscher untersuchten den Wechsel der Mittelcapacität der Jungen unter dem Einfluss verschiedener Körperstellungen. Bohr und sein Schüler Hasselbalch haben dieses Thema in neuerer Zeit (1907) wieder aufgenommen. Nach ihren übereinstimmenden Untersuchungen steht es fest, dass beim Lebergang aus der stehenden oder sitzenden Stellung in die liegende die Mittelcapacität der Lungen beträchtlich zurückgeht. Allerdings erwies sich die Voraussetzung Bohr's als irrig, dass die Totalcapacität der Lungen eine unter allen Versuchsbedingungen gleichbleibende Grösse sei. Hasselbalch konnte vielmehr zeigen, dass beim Uebergang aus dem Stehen zum Liegen die Totalcapacität bis zu $8 / 4$ Liter abnimmt. Ohne dass die Residualluft sich irgendwie wesentlich ändert, nimmt dabei die Reserveluft erheblich ab.

Aber auch bei anderen Versuchen ändert sich, wie wir später sehen werden, die 'lotalcapacität.

1) Vitalcapacität: Aus der normalen ruhigen Athmung, der Mittellage heraus, wird extrem eingeathmet: Complementärluft, oder extrem ausgeathmet: Reserveluft. Vitalcapacität also $=$ Volumdifferenz zwischen der äussersten Inspirations- und der äussersten Exspirationsstellung dor Lunge. Residualluft ist die nach tiefster Exspiration noch in der Lunge befindliche Luft. Mitteloapacität = Volumen der Lungen in der Mittellage, also bei ruhiger, normaler Fin- und Ausathmung. Totalcapacität $=$ Vitalcapacität plus Residualluft. Érhöhung der Mittellage bedeutet 'Lunahme der Mittelcapacität, Lungenerweiterung, Lungenblähung, Volumen pulmonum auctum, normales temporäres Emphysem (Bohr). 
Das bedeutet, dass die Untersuchungen Bohr's über die Veränderung des Lungenvolumens unter den verschiedensten Einflüssen nur approximative Werthe geben, da Bohr den Werth für die Nittelcapacität der Lungen aus der ein für alle Male bestimmten Totalcapacität minus der jeweiligen Complementärluft errechnete.

Ueberraschend ist übrigens diese Beobachtung Hasselbalch's nicht, weiss man doch schon lange, dass beim Uebergang vom Stehen zum Jiegen der vordere Leberrand percussorisch um $2,6 \mathrm{~cm}$ cranialwärts rückt. Ilan nahm dementsprechend ein Hineinsinken der Leber in den Thoraxraum an.

Bestätigt wird diese Auffassung durch die Röntgenuntersuchung (Holzknecht, II fbauer, Levy-Dorn). Die Zwerchfellinie steht bei dorsoventraler Durchleuchtung in aufrechter Stellung rechts am oberen Rand der 5. Rippe, im Liegen am unteren Rand der 4. Rippe. Nach 10 . 15 . Winuten langem Jiegen ist dann, wic ich mich mittclst der Orthodiagraphie des Zwerchfells überzeugen konnte, die Zwerchfellkuppe, d. h. die Juber, noch mehr cranialwärts getreten. Andererseits aber gelang es mir nicht, bei einer Versuchsperson im Stehen nach raschem Anfüllen des rorher leeren Magens mit 1500 cem Wasser ein Tiefertreten der Zwerchfellschattengrenze und damit eine gewisse Zunahme des Lungenvolumens zu constatiren.

Bei frontaler Strahlenrichtung findet man ausserdem, dass die nach hinten gelegenen $/$ werchfelltheile im Liegen noch höher gedrängt werden als die vorderen. Es werden also wohl beim lebergang vom Stehen zum Liegen bei dorsoventraler Strahlenriehtung andere, mehr nach der Wirbelsäule zu gelegene Zwerchfellpunkte schattenrandständig.

Es dürfte hier, ehe wir weitergehen, eine kurze Kritik der Methoden am Platze sein, mit welchen wir uns über das Jungenvolumen, d. h. die Lungencapacität, zn orientiren suchen.

Die Spirometrie giebt nur dann sicheren Aufschluss, wenn man, wic Ilasselbalch und Siebeck, bei jedem einzelnen Versueh neben der Vitalcapacität auch die Residualluft experimentell bestimmt und nicht eine der Grössen aus früheren Untersuchungen zur Berechnung heranzieht.

Unser llauptinteresse gilt natürlich der jeweiligen I.ungemmittellage, d. h. wir wollen wissen, welche Juftmenge in der Junge sich befindet, während der Entersuchte ruhig, unter Vermeidung körperlicher Arbeit, aus und ein athmet. Die Vitalcapacität, das Product extremer Muskelanstrengung, ist von zu vielen zufäligen Factoren abhängiy. Dementsprechend hat auch die Totalcapacität im einzelnen Versuch für uns ein weniger grosses Interesse.

So gut wie alle bisherigen Beohachtungen über vorübergehende Lungenblähung sind auf percussorischem Wege gewonnen.

Jass man mit Recht aus dem Tiefertreten der unteren Lungengrenzen und einem gewissen hypersonoren Sehall auf eine Zunahme des Thoraxinnenraumes und damit der Lungencapacitä schloss, zeigt die Röntgendurehleuchtung.

Wir sehen bei percussorischem Tiefstand der unteren Lungengrenze 
im Röntgenlicht Erwoiterung der Complementärräume sowie Tiefertreten und Abflachung der Zwerchfellkuppen.

Man muss sich hüten, allein aus dem Tiefertreten der Zwerchfelllinie bei der üblichen dorsoventralen Durchleuchtung auf den Eintritt einer Lungenblähung zu schliessen, auch wenn die Linie orthodiagraphisch aufgenommen wurde. Lungenblähung darf nur dann diagnosticirt werden, weun die Durchleuchtung auch in einer zweiten Strahlenrichtung ein Tiefertreten der ganzen Zwerchfelllinie ergiebt.

Wie die Percussion und Röntgendurchleuchtung, so geben auch Pneumographie und Stethographie (Bert) keinen Aufschluss über die absolute Grösse des jeweiligen Lungenvolumens, sondern dienen nur dem Yergleich bezüglich der Form und Ausdehnung des Thorax und damit der Lungen vor und nach kurzfristigen Lungenblähungen.

Bei dem Wechsel der Körperlage handelte es sich um eine rein passive Veränderung des "mittleren Lungenvolumens" durch Einengung des Thoraxinnenraumes vom Abdomen her.

Wir betrachten nun im Folgenden wie weit die willkürliche und die unwillkürliche Aenderung der Athmungsweise einen Wechsel der Lungencapacität hervorzurufen vermag.

Schon wenn wir den Thorax in einer gewissen Inspirationsstellung willkürlich fixiren und nun in dieser Stellung ruhig aus und ein athmen, so athmen wir mit erhöhter Lungen-Mittellage, d. h. relativ erweiterten Lungen. Ebenso nimmt die Thorax- und Lungencapacität wesentlich zu, wenn wir durch energische Extension der Brustwirbelsäule aus bequemer in militärisch stramme Haltung übergehen.

Körperanstrengungen aller Art führen zu u n willk ürlicher Verstärkung der Athmung. Führen sie dabei auch zu einer Veränderung der Lungenmittellage?

Moritz notirt (1902) in einer Arbeit über orthodiagraphische Herzuntersuchung bei einem Manne, der $33 \mathrm{~km}$ in 1 Stunde 26 Minuten auf dem Rade gefahren und dabei stark dyspnoisch geworden war, einen Zwerchfelltiefstand. In der Arbeit von Moritz und Dietlen (1908) über Herzuntersuchungen von Radfahrern, die die Strecke Leipzig-Strassburg in 2 Tagen zurückgelegt hatten, steht jedoch nichts von Lungenblähung; auch weisen die Orthodiagramme keinen Zwerchfelltiefstand auf. Allerdings wurden die orthodiagraphischen Aufnahmen frühestens 10 -20 Minuten nach Beendigung der Fahrt angefertigt.

Schieffer (1906) wiederum, ein Schüler von Moritz, fand bei einem Radfahrer nach einer Tour von $40 \mathrm{~km}$ in 2 Stunden, noch dazu bei schlechten Wegen, ein deutliches Volumen pulmonum auctum, was auch aus der Differenz der beigegebenen Orthodiagramme vor und nach der Radtour hervorgeht.

Ebenso will Schott-Nauheim in 2 Arbeiten aus den Jahren 1890 und 1908 bei Ringkämpfern ein Volumen pulmonum auctum unter Herabrücken des $\mathrm{Z}$ werchfelles beobachtet haben.

Kienböck, sowie Selig und Beck (1907) vermissen dagegen bei Wettschwimmern Zeichen von Lungenblähung bezw. Zwerchfelltiefstand. 
Allerdings hat Moritz ihre Orthodiagramme mit Recht für sehr ungenau erklärt.

Ferner finden wir in einer Arbeit von Albu und Caspari (1903) über die Resultate eines Wettmarsches die Angabe, dass bei einem der Wettgänger die Lungenlebergrenze in der Mamillarlinie an der 8. Rippe gestanden habe.

Die Angaben dieser Autoren sind natürlich insofern besonders werthvoll, als man die Befunde über das Volumen pulmonum auctum ohne jede Voreingenommenheit als mehr oder weniger zufällige Nebenbefunde notirte.

Diese Beobachtungen zeigen, dass angestrengte Körperarbeit zu Lungenblähung führen kann. Eine Bestätigung bringen die einwandfreien spirometrischen Untersuchungen von Durig (1903). Durig fand bei 2 gesunden Personen nach einer anstrengenden 19 stündigen Gebirgswanderung eine Vermehrung der Residualluft um $200 \mathrm{ccm}$, die sogar bei einem der beiden Männer 2 Tage lang anhielt.

Ferner fand Hasselbalch 1908 bei seinen daraufhin angestellten Arbeitsversuchen ausnahmslos eine Vermehrung der Residualluft um 300 bis $500 \mathrm{ccm}$, daneben Gleichbleiben bezw. Zunehmen der Reserveluft; das bedeutet also eine Erhöhung der Mittelcapacität um 200 bis $700 \mathrm{com}$. Gleichzeitig beobachtete er eine wesentliche Abnahme der Complementäriuft und dadurch der Vitalcapacität um 100-300 cem, jedoch eine Zunahme der Totalcapacität um $100-410 \mathrm{ccm}$ in Folge der Zunahme der Residualluft.

Bei der durch einen Schnellauf hervorgerufenen gewaltigen Steigerung der Athmungsthätigkeit kommt der Thorax in eine mehr oder weniger ausgesprochene Inspirationsstellung. Wir empfinden das schon selbst an der Unfähigkeit, so tief wie vorher einzuathmen. Aber die Grenze der tiefsten Inspiration ist trotzdem erweitert, so dass der Thorax bezw. die Lunge in den Momenten extrem forcirter Einathmung mehr Luft enthalten, als vor dem Lauf unter denselben Bedingungen.

Bei 2 von den H.'schen Versuchen sehen wir bemerkenswerther Weise die Lungenblähung nach 10 Minuten wieder verschwinden, während Athemgrösse und Pulszahl noch nicht zur Norm zurückgekehrt sind. Bei zwei weiteren Versuchen ist über die Dauer der Blähung nichts angegeben.

Ausser dieser vorübergehenden Lungendehnung während und nach Körperanstrengung giebt es dann noch die allmähliche Zunahme der Thorax- und Lungencapacität durch Uebung. Die Residuallult bleibt davon unberührt. Es nimmt die Fähigkeit der tiefen Ein- und Ausathmung entsprechend zu. Bei einer von Hasselbalch controlirten Versuchsperson nahmen im Laufe eines halben Jahres durch entsprechende Uebung Mittel- und Totalcapacität des Thorax und der Lungen um rund $1 / 2$ Liter zu. Sportsmänner mit zum Theil enormer Totalcapacität (7000 cem) haben auffallend niedere Residualluft, wohl weil sie gelernt haben, ausgiebiger ihre auxiliaren Exspirationsmuskeln anzuwenden.

Ferner haben dann auch Hofbauer und Holzknecht ausgedehnte 
röntgologische Untersuchungen angestellt über die Entstehung der Lungenblähung. Sie skizzirten den Zwerchfellstand bei normaler und vertiefter In- und Exspiration auf den Fluorescenzschirm und beobachteten dabei, dass der exspiratorische Zwerchfollstand bei vertiefter Athmung mehr caudalwärts steht als bei der gewöhnlichen ruhigen Athmung. Auf Grund dieser Beobachtung schreiben sie wie folgt: "Wenn man entweder auf Befehl oder in Folge des Gefühls von Lufthunger die Athmung vertiefen will, so vollzieht sich das so, dass in beinahe allen Fällen nur die Einathmung an Stärke gewinnt und die Ausathmung sogar in vielen Fällen nicht nur nicht gesteigert, sondern sogar in ihrer Intensität gegenüber der Norm herabgesetzt wird. Dieser unzweckmässige Mechanismus liesse es begreiflich erscheinen, wenn in Folge einer solchen geänderten $\Lambda$ themmechanik sich consecutiv Lungenblähung einstellte. Es wird, so schreiben sie, uns dadurch geradezu Einblick gewährt in die pathologische Physiologie des Emphysems."

\section{Eigene Versuche.}

\section{A. Unwillkürliche Vertiefung der Athmung in Folge Körper- anstrengung (Lauf).}

Hierbei haben wir manchmal die exspiratorische Zwerchfellschattengrenze nach dem Lauf etwas tiefer gefunden als vorher, also Volumen pulmonum auctum constatiren können.

Wir haben übrigens zwecks Aufzeichnung der Zwerchfellgrenzen keine unserer Versuchspersonen den Athem, wenn auch noch so kurz, anhalten lassen; daher ist die Markirung der Zwerchfellgrenze nach dem Lauf bei der jagenden Athmung schwierig.

\section{B. Willkürliche Athemvertiefung.}

1. In 12 Versuchen fanden wir die 7werchfellschattengrenze in tiefer Einathmungsstellung wenig tiefer, ja gelegentlich höher als am Ende der ruhigen Einathmung in Folge der beträchtlichen inspiratorischen IIebung des Thorax und damit der Zwerehfellansätze.

2. Die Zwerchfellschattengrenze stand in der tiefen Exspirationsstellung häufig etwas höher als bei der ruhigen Ausathmung, noch öfter gleich hoch, sehr selten unterhalb.

3. Wenn es durch willkürliche Vertiefung der Athmung zu einer echten sich durch Zwerchfelltiefstand charakterisirenden Lungenblähung käme, so müsste jener auch noch nach dem Uebergang aus der vertieften in dic ruhige $A$ thmung nachweisbar sein.

Unsere Versuche zeigen jedoch, dass der exspiratorische Zwerchfellstand nach Aufhören der vertieften Athmung mit dem bei ruhiger Athmung vor Beginn der tiefen $\Lambda$ thmung übereinstimmt. Abweichungen von diesem Befund fallen in die Versuchsfehlerzone.

\section{Willkürlich möglichst schnelles und tiefes Athmen.}

Bei diesen Versuchen fanden wir einige Male ein geringes Tiefertreten, aber ebenso oft ein Gleichbleiben des exspiratorischen Standes der Zwcrehfellschattengrenzc. 
Uebrigens haben wir bei unseren Untersuchungen des Zwerchfellstandes mit dem Grödel'schen Orthodiagraphen trotz aller erdenklichen Cautelen, Anbringung von Controlbleimarken an Brust und Untersuchungstisch, Markirung der ganzen Thoraxumrandung vor und nach der tiefen Athmung, bei In- und Exspiration gesehen, dass diese Methode nicht dazu geeignet ist, Schwankungen des mittleren Lungenvolumens unter oben genannten Versuchsbedingungen zuverlässig anzugeben. Wir vermögen mit dieser Methode eben nur die schattenrandständigen Theile der Brustraumwände zu markiren und das genügt nicht zu einer exacten Volumbestimmung des Thoraxinhalts.

Ausser den orthodiagraphischen Untersuchungen des Zwerchfellstandes haben wir auch noch zahlreiche pneumographische und stethographische Curven mit Apparaten nach P. Bert aufgenommen: 1. nach Körperanstrengung, 2. bei willkürlich vertiefter ruhiger Athmung und 3. bei willkürlich beschleunigter Athmung.

Es wurden gleichzeitig zwei Curven geschrieben, die obere giebt die Schwankungen des Thoraxdurchmessers wieder in Höhe der Mamillen, die andere in Höhe des unteren Endes des Processus xyphoideus.

Betont muss dabei werden, dass aus den Curven die absolute Athemtiefe nicht gemessen werden kann, da die Athemausschläge der Curve ja nicht allein von der Grösse der Thoraxbewegung abhängig sind, sondern auch von dem mit jeder Aenderung der Spannung wechselnden Elastizitätsmodul der Gummimembranen der Aufnahme- und Registrirtrommeln. Messen lässt sich nur das Verhältniss der Athmungstiefe zur Athmungsdauer als Verhältniss des Ordinaten- zum Abscissenwerth.

Die heifolgenden Curven (Taf. XII) stellen die Registrirung der Bewegung eines bezw. zweier Punkte der Brustwand unter Zuhülfenahme der Luftübertragung dar. Der Apparat entsprieht etwa dem ron P. Bert (Jeçons sur la physiologie comparé de la respiration. Paris 1870) und besteht im Wesentlichen aus einer Aufnahruetrommel, deren Pelotte der Brustwand anliegt und einer Registrirtrommel mit Sehreibhebel. Der aulsteigende Schenkel der Curve des Stethogramms entspricht der Inder absteigende der Exspiration. Die anderen Curven geben die Veränderung zweier Querschnitte des Brustkorbes wieder. Sie sind aufgenommen mit von P. Bert modificirten Thorakographen Marey's, kurzen Metallcylindern, die an beiden Enden mit Gummimembranen verschlossen, durch Bänder horizontal um den Brustkorb befestigt sind. Die Registrirung geschieht ebenfalls durch Luftübertragung. Die inspiratorische Brustkorbdehnung zieht die Membranen, die mit aufgeklebten Häkchen an die Bänder befestigt sind, aus dem Cylinder heraus. Der Luftverdünnung entsprechend sinkt der Schreibhebel der Registrirtrommel bei der Inspiration und umgekehrt. Die so gewonnenen Curven heissen Pneumogramme.

\section{Athemcurve nach Körperaustrengnng.}

Die Versuchsperson liegt auf der Erde und macht rasche Flexionsbewegungen in Hüften und Kniegelenken, die Füsse sind mit Sandsäcken beschwert. Die Versuchsperson arbeitet bis $\Lambda$ themnoth eintritt.

Wir sehen an Figur I, besonders wenn man die Fusspunkte der einzelnen Inspirationen und Exspirationen miteinander verbindet, wie 
nach Abschluss der Arbeit die inspiratorischen Ausschläge, also die Inspirationsbewegungen des Thorax wesentlich rascher an Grösse abnehmen als die exspiratorischen; das bedeutet, dass eine Zeit lang mehr Luft aus den Lungen entfernt, als ihr zugeführt wird, dass also eine Ueberfüllung der Lungen mit Luft bestanden hatte.

\section{Willkürlich vertiefte (Fig. III a, IIIb)}

3. Willkürlich beschleunigte Athmung (Fig. II).

Um zu prüfen, wer bei der Aufforderung, tief zu athmen, nur die Einathmung vertieft, dazu hätten wir diese Untersuchungen mit Röntgenlicht und Pneumographen nicht nöthig gehabt. Beobachten wir doch bei der Lungenuntersuchung am Krankenbett, dass eine grosse Anzahl von Menschen, energisch und wiederholt aufgefordert, tief zu athmen, ihre Lungen mit Luft vollpumpen, so dass wir ihnen dann erst zeigen müssen, wie man ausathmet. Eine derartige Luftüberfüllung hat keinerlei Bedeutung. Sie ist nach 2-3 Athemzügen wieder ausgeglichen.

Wir wollten vielmehr untersuchen, ob es bei tiefer Ein- und Ausathmung zu einer inspiratorischen Ueberdehnung der unteren Lungenabschnitte im Sinne Tendeloo's kommt und wie lange eine eventuelle Volumzunahme der unteren Thoraxpartien bestehen bleibt.

1. Wie Curve III a zeigt, wird bei der ersten vertieften Exspiration nicht nur die Luft wieder aus der Lunge entfernt, welche die erste vertiefte Inspiration ihr zuführte, sondern es wird ausserdem noch um ebensoviel mehr Luft ausgeathmet, als die.erste vertiefte Einathmung die normale Inspirationsgrösse übertraf.

2. Manchmal sehen wir jedoch auch (Fig. III b), dass die Exspirationen nur die Luft der vertieften Inspirationen wieder aus der Lunge entfernen. Dabei erreicht aber der exspiratorische Schenkel der Curve mindestens die gleiche Höhe, die er bei normaler Athmung, also vor Beginn der Athemvertiefung, hatte. Der Verlauf der Gesammtcurve ist dabei absolut horizontal. Er müsste bogen- bezw. staffelförmig sein, wie wir ihn auf andern Curven noch sehen werden, wenn bei jeder folgenden Exspiration ein Bruchtheil der Luft der vorangegangenen Inspiration im Thorax zurückbleiben würde.

Es demonstriren also auch die Pneumo-bezw. Stethogramme, dass bei willkürlich vertieftem bezw. beschleunigtem Ein-und Ausathmen eine Lungenblähung nicht eintritt.

Auch Bohr hat Leute möglichst schnell und tief athmen lassen und fand dabei auf spirometrischem Wege sogar eine geringe Abnahme des mittleren Lungenvolumens.

Andererseits spricht Jamin's Beobachtung nicht gegen uns. Jamin sah bei einer stundenlang anhaltenden angestrengten und enorm besehleunigten Athmung eines Hysterikers Tiefstand beider Zwerchfellkuppen im Röntgenbild. Die Lungenblähung bildete sich mit Aufhören des Erregungszustandes alsbald wieder zurück. Ein derartig schwerer Erregungszustand der Respirationscentren führt complicierte Athembedingungen ein, die wir erst später analysieren werden. 
Ueber den Einfluss sauerstoffarmer und $\mathrm{CO}_{2}$-reicher Luft nahm Bohr Untersuchungen vor auf die Grösse des Lungenvolumens. Er konnte dabei feststellen, dass, wenn die Finathemluft statt der normalen 21 pCt. nur 11-12 pCt. $\mathrm{O}_{2}$ enthielt, eine Zunahme der Mittelcapacität eintritt, die im Liegen bis 0,4 Liter beträgt.

Die Zunahme des mittleren Lungenvolumens, die bei Finathmung $\mathrm{CO}_{2}$-haltiger $\mathrm{L}_{\mathrm{uft}}$ eintritt, jst recht deutlich und beträgt, wenn der $\mathrm{CO}_{2}$-Gehalt $(7,6 \mathrm{pCt}$.) die Koblensäureausscheidung aus dem Blut in der Lunge verhindert, etwa 1 Liter. Aber schon bei der Einathmung einer IJuft mit niedrigem $\mathrm{CO}_{2^{2}}$-Gehalt $(4,5 \mathrm{pC}$ t. $)$ tritt, während die $\mathrm{CO}_{2}-\mathrm{A}$ usscheidung noch völlig normal ist, eine Zunahme der Stärke der Athemzüge auf das Doppelte cin (Speck).

Bohr bestätigt somit die Angrabe Gad's, dass bei vermehrter Venositat des Blutes auch in tiefster Exspirationsstellung mehr luft als normal in den langen bleibe.

Die Erkenntniss, dass durch Veränderung der ehenischen Zusammensetzung der Finathemluft nicht nur die Frequenz und Stärke der Athembewegungen, sondern auch die Grösse des Lungenvolumens Aenderungen erleiden, legte es nahe, diesbezügliche Untersucbungen boi Herzkranken vorzunehmen, besonders da man sich zu der Annahme berechtigt glaubte, dass die Aenderung der Blutheschaffenheit im $\Lambda$ themcentrum selbst die Veranlassung zu dieser Volumänderung der Lunge sei.

Die einzigen Arbeiten auf diesem Gebiete verdanken wir knbow. Jir fand, dass bei 3 schwer IIerzkranken sehon in Körperrube die Mittelcapacität der Lunge grösser war als die böchste Mitteleapacität bej 16 normalen Controlpersonen. Dabei bestand, zum Theil einem niedrigen respiratorischen Stofiwechsol entsprechend, keine wesertliche Vermehrung der Respirations-Frequenz noch -Stärke, sondern eine langsime, relativ tiefe Athmung, jedoch mit dem Gefühl einer gewissen Athemnoth.

Im Alloemeinen reagiren leicht Herzkranke auf mässige körperliche Anstrengung wie Gesunde. Bei einer Patientin jedocb führte eine derartige Anstrengung, allerdings zusammen mit einer gemüthlichen Alteration, zu einer sehr bedeutenden Lungenblähung obne jede Aenderung der Respirationsform, aber mit jenem Gefühl der Athemnoth.

Besonders hochgradigen Formen der Lungenblähung begegnen wir unter dem Bild der paroxysmalen Iyspmoe, sowohl bei der Neurose der Hermerven: dem essentiellen Asthma cardialo, als bei organischen Nieren-, Geläss- und Herzkrankheiten. A ber auch beim Asthma bronchiale, dieser Neurose der lungennerven, komnt dic Lungenblähung unter denselben Erscheinungen za Stande, so dass wir alle zusammen hier abhandeln können.

Der einzelne Anfall beginut nit tiefen, angestrengten Inspirationsbewegungen. Inspiration und Lixspiration sind Anfangs vorlängert, besonders aber die Exspiration. Der Kranko nimmt sitzend oder stehend cine Haltung an, die ihm dir ersehwerte Inspiration möglichst erleichtert und ihm hilft, die Brust in Inspirationsstellung zu halten. Inspirirt wird sichtlich unter grosser Anstrengung; die Inspirationsmuskeln bleiben aber vielfach auch während der lixspiration in einer gewissen Spannung. 
Häufig auch beobachtet man tonischen Krampf der auxiliären Inspirationsmuskeln. Die Athmung wird erst dann oberflächlicher, wenn der Brustkorb maximal gedehnt und die Lungen durch die tiefen Inspirationen gebläht sind.

Ueber Veränderungen des Lungenvolumens durch sensible Reizungen ist manches bekannt. Hasselbalch hat den Einfluss wechselnder Iuftund Wassertemperaturen studirt. Iseider sind bei den diesbezüglichen Versuchen wieder nur Vitalcapacitätsbestimmungen gemacht und ist auf die directe Bestimmung der Residualluft und 'Totalcapacität verzichtet. Trotzdem darf man mit dem Autor schliessen, dass durch die Einwirkung kalter Juft bezw. kalten Wassers auf den bis dahin warm gehaltenen Körper eine eventuell nicht ganz unbedeutende Vermehrung der Lungenmittelcapacität eintritt.

Dieser Befund einer vorübergehenden Lungenerweiterung wird bestätigt durch Versuche, die Beck und Dohan mit heissen und kalten Büdern anstellten. Bei diesen Versuchen stellten die beiden Autoren häufig eine "Lungenblähung" mit Zwerchfelltiefstand fest.

Im Thierexperiment hat Einthoven die Veränderung des Athemdruckes durch periphere und centrale Reizung des Lungennerven (Vagus) untersucht. Da nun, wie er sich überzeugte, mit der Erhöhung des $\Lambda$ themdruckes regelmässig eine Zunahme des Lungenvolumens sich einstellte, so haben Einthoven's Resultate auch für uns grösstes Interesse.

Periphere elektrische Vagusreizung führte stets $z u$ erheblicher Lungenblähung. Bemerkenswerth ist, dass es bei Reizung des centralen Endes des einen durchschnittenen Vagus nicht gelang, Athemdruckerhöhung und Lungenblähung zu erzeugen.

Centrale Ischiadicusreizung führte nicht immer zur Athemdruckerhöhung. Diese trat jedoch ein bei einer anderen Methode der reflectorischen Vaguserregung, dem Einblasen von $\mathrm{SO}_{2}$ in die Nasenlöcher. Beim Einathmen reiner $\mathrm{CO}_{2}$ trat stets starke Lungenblähung und Blutdruckstejgerung ein. Einathmung eines $\mathrm{CO}_{2}$-Gemisches führte ebenfalls regelmässig $z u$ sehr beträchtlicher Athemdruckerhöhung, die sofort verschwand, wenn die Vagi durchschnitten wurden.

Aus der menschlichen Pathologie ist bekannt die recht erhebliche Lungenblähung bei Angstzuständen Geisteskranker (Griesinger, Ziertmann).

Die Athmung der Kranken ist im Anfall hauptsächlich costal, angestrengt, dabei frequent und wegen der Inspirationsstellung des Thorax oberflächlich. Durch die ausserordentliche Unruhe der Kranken waren eingehendere Ĺntersuchungen unmöglich. Genauere Beobachtungen über das Einsetzen der Lungenblähung fehlen. Die Jungengrenzen standen vorne rechts an der 8.--9. Rippe. Die absolute Herzdämpfung war nicht aufgehoben. Ziertmann constatirt ausserdem noch Kälte und Blässe der Extremitäten und des Gesichts, weite Pupillen und häufige Schweissausbrüche.

Weiterhin hat Zuelzer zweimal plötzlich einsetzende Anfälle heftiger I ungenblähung mit paroxysmaler Dyspnoe beobachtet bei Leuten mit Störungen in den Verdauungsorganen (Obstipation). Bei 26 
weiteren Fällen sah er dasselbe Bild nur in wesentlich milderer Form. 8 von diesen Kranken litten an hartnäckiger Obstipation. Bei den acuten Fällen beseitigt die Ausschaltung des Vagus durch $1 \mathrm{mg}$ Atropin die Anfälle prompt.

Ferner haben Tuczek und Kredel klinische Beobachtungen publicirt von anfallsweise auftretender Lungenblähung mit Pulsbeschleunigung. Die Ursache suchten sie in einer gleichzeitigen Reizung der Lungen- und Lähmung der Herzfasern des Vagus. Der Reiz auf den Vagus sollte nach ihrer Ansicht von an- und abschwellenden tuberkulösen Bronchialdrüsen ausgehen. Mit dem Aufhören des Angstzustandes verschwand auch regelmässig die Lungenerweiterung vollstündig.

Wir kommen nunmehr zor Lungenblähung bei primärer Veränderung der Athomwege und -öffnungen. Wir könren eine derartige Blähung beobachten, wenn wir durch ein entsprechend dünnes Glasrohr ein- und ausathmen oder bei unbehinderter Einathmung durch ein solches Rohr ausathmen lassen. Dann tritt nach einiger Zeit eine typische Lungenblähung auf. Es ist dies ein Vorgang, der sich z. B. einstellt, wenn ein Ungeübter Hoboe bläst.

Um auf die Pathologie überzugehen, so begegnen wir der temporären Lungonblähung recht häufig bei den Stenosen der oberen Luftwege, wie sie durch Croup, Diphtherie, Glottisödem und Stimmbandpolypen gelegentlich entstehen. $O b$ es zur Lungenblähung kommt, hängt ganz von Grad, Sitz und Dauer der Verengerung, sowie von der Schnelligkeit des Eintritts derselben ab. Je nachdem mehr die In- oder Exspiration erschwert ist, sind ganz verschiedene Lungentheile gebläht. Am häufigsten ist die Lungenblähung inspiratorisch-exspiratorischen Charakters (Keuchhusten, Bronchiolitis). Rein exspiratorische Lungenblähung ist aber auch nichts Seltenes. Wir lernen sie kennen z. B. bei Laryngitis ohne Verengerung und bei Bronchitis, also bei Krankheiten, die mit starkem Husten einhergehen.

Die schwere $\mathrm{CO}_{2}$-Vergiftung des Athemeentrums und die Todesangst, wie sie die Embolien der Art. pulmonalis bedingen, führen durch heftigste Inspirationen und wohl gleichzeitigen Bronchialmuskelkrampf zu schwerer inspiratorischer Lungenblähung.

Ueberblicken wir die Reihe, so sehen wir die Lungenblähung unter pathologischen Bedingungen genau so auftreten wie im Experiment.

Wir beginnen mit den Lungenblähungen, die hervorgerufen sind durch willkürliche Becinflussung der Ein- und Ausathmung bezw. durch krankhafte Stenosen der oberen und tieferen Luftwege.

Bert hat ähnliche Versuche angestellt. Jedoch registrirte er dabei nur die respiratorischen Luftdruckschwankungen in einer geschlossenen Flasche. Der grosse Nachtheil dieser Methode, auf den 'T'endeloo mit Recht hinweist, ist, dass bei Stenoseversuchen die Grösse der respiratorischen Brustwandbewegungen viel grösseren Schwankungen unterworfen ist, als die aufgezeichneten Luftdruckschwankungen anzeigen. „So darf man z. B. aus dem gleichbleibenden inspiratorischen Luftdruck nicht schliessen, dass die Lungen sich nicht ungewöhnlich vergrössern." 
Die Curve IV ist gewonnen bei gleichmässiger Erschwerung der In- und Exspiration.. Es wurde bei verschlussener Nase durch ein Glasrohr geathmet, dessen vordere Oeffnung auf etwa $2 \mathrm{~mm}$ verengt war. Bei darauf gerichteten Vorversuchen hatte sich gezeigt, dass diesem Grad der Verengerung die Ein- und Ausathemkräfte nach relativ kurzer Zeit nicht mehr gewachsen sind. Nasenverschluss und Glasrohr werden entfernt, sobald die Athemnoth peinigend geworden ist.

Als erste Folge der Athmungserschwerung sehen wir eine Verlangsamung der Athemfrequenz hauptsächlich auf Kosten der Exspiration, sowie eine Vertiefung beider Athemphasen. $\mathrm{Da}$ jedoch die einzelnen Inspirationen die Exspirationen an Grösse übertreffen, so ist der Curvenverlauf ein langsam staffelförmig ansteigender. Gleichzeitig mit heftigem Lufthunger tritt dann eine Beschleunigung der Athemzüge ein. Bald jedoch zwingt die übermächtige Beklommenheit zur Freigabe der Athemöffnungen. Jetzt erfolgen eine Reihe ausgiebiger Athemzüge. Die Rückkehr zum normalen Thorax- und damit Lungenvolumen sehen wir langsam vor sich gehen. Wir beobachten, dass nach Beseitigung der Stenose der Thorax meist eine Reihe energischer A usathembewegungen macht, welche die entsprechenden. Einathembewegungen an Grösse nicht unwesentlich übertreffen. Nach 10-15 Athemzügen bezw. nach ca. $1 / 2$ Minute ist die Curve wieder auf ihrem früheren Niveau angelangt und die Grösse der Athemzüge zur Norm zurückgekehrt.

10 weitere Versuche dieser Anordnung haben denselben Curvenverlauf ergeben. Wurden bei einem Versuch Glasröhre und Nasenverschluss im Moment des Beginns der erschwerten Finathmung entfernt, so erfolgte wegen des Lufthungers zuerst eine vertiefte Einathmung vor der Serie der entlastenden Ausathembewegungen.

Der Eintritt einer Jungenblähung hängt natürlich auch bei arteficieller Verengerung der Athemöffnung vom Grad, von der Dauer und der Schnelligkeit des Eintritts der Stenose ab. Speciell noch stärkere Verengerungen als die in unseren Versuchen führen zu gleichzeitiger Insufficienz der Aus- und Einathemkräfte.

Curve $V$ stellt die Veränderungen des Thorax- und Lungenvolumens dar bei und nach erschwerter Ausathmung ohne Behinderung der Einathmung. Die Ausathmung geschieht ebenfalls durch ein Glasrohr mit etwa $2 \mathrm{~mm}$ grosser Oeffnung. Mit der Erschwerung der Ausathmung sehen wir Anfangs eine beträchtliche Verlangsamung und Vertiefung der Athmung. Bald jedoch constatirt man an der Curve das Ueberwiegen der Inspirations- über die Exspirationsgrösse. Unter staffelförmigem Ansteigen der Curve wird der Thorax gedehnt und nun nimmt auch die Athemtiefe rasch ab. Die vermuthlich compensatorische Beschleunigung der Athemfrequenz vermag das Gefühl heftiger Beklemmung nicht zu bannen und so müssen nach etwa 12 Athemzügen Glasröhre und Nasenklemme entfernt werden.

Die Entspannung des Thorax und der Rückgang der Lungenblähung: treten ein durch eine Reihe energischer Ausathembewegungen, die wiederum die zugehörigen Inspirationen beträchtlich an Grösse übertreffen.

Es ist zu beachten, dass der horizontal verlaufende Theil des der 
Exspiration entsprechenden absteigenden Curvenschenkels zur Exspiration selbst gehört, also eine Verlängerung dieses Schenkels bedeutet und nicht etwa einer Athempause entspricht. Die Rückkehr der Curve zum früheren Niveau und der Athemgrösse zur normalen ist nach 1 bis 2 Minuten erfolgt.

In der unteren der beiden Curven, welche die respiratorische Bewegung des unteren Sternaltheils wiedergiebt, findet sich auf dem der Exspiration entsprechend absteigenden Curvenschenkel während des "Stenoseathmens" je eine grosse Zacke. Sie entspricht der durch Contraction speciell der Muskeln der Unterbauchgegend hervorgerufenen Vorwölbung des Epigastriums und der unteren Sternumpartien zu Beginn der erschwerten Exspiration.

Zahlreiche Untersuchungen mit Bandmaass und Kyrtometer, die ich während und nach Versuchen mit erschwerter Einathmung bei unbehinderter Ausathmung vornehmen liess, ergaben fast ausnahmslos eine Zunahme des unteren Thoraxumfanges um mehrere Centimeter und eine Abnahme der oberen Thoraxpartien um etwa ebenso viel.

So gross die Zahl der bisher besprochenen Fälle von Lungenblähung, so mannigfach die Art ihrer Entstehung und die Auffassung über ihre Bedentung.

Warum wir - auch thierexperimentell - nur bei bestimmten Graden von Verengerung der oberen Athemwege Iungenblähung beobachten, hat T'endeloo zu erklären versucht, um dadurch die Widersprüche in den Angaben früherer Autoren zu beseitigen. (Bert, Marey, Conheim, Köhler.)

Welche Factoren im Einzelnen die Aenderung der Athemtiefe und -Frequenz während des Versuchs bewirken, ist noch nähor zu untersuchen. Es handelt sich dabei um psychische Einflüsse, periphere Nervenbesonders Vaguserregung und Aenderung der Blutgase.

In sehr interessanten Arbeiten über die Dyspnoe durch Stenose der Luftwege stellten Morawitz und Siebeck fest, dass die erschwerte angestrengte Athmung (Dyspnoe) durch keinen der chemischen Athemreize hervorgerufen werde. Erst, wenn das Gefühl lebhafter Athemnoth sich einstellt, fanden sie die $\mathrm{CO}_{2}$-Spannung der Alveolarluft erhöht. Uebrigens hat schon Sänger 1906 betont, dass das subjective und objective Luftbedürfniss keineswegs in einem unveränderlichen Stärkeverhältniss stehen.

Ferner weist Siebeck als Resultat seiner Versuche auf den höchst wahrscheinlichen Zusammenhang der Vergrösserung des Athemvolumens und Erhöhung der Mittellage hin.

Auf Grund unserer Untersuchungen sind wir der Auffassung, dass diese Form der Lungenblähung in erster Linie mechanisch zu erklären sei und zwar aus einem Zurückbleiben der exspiratorischen Lungenverkleinerung hinter der inspiratorischen Erweiterung. Dies geschieht aus zwei Gründen.

Stenoseversuche an uns selbst haben uns gezeigt, welchen Einfluss die Psyche auf den Eintritt der Lungenblähung ausübt. Es ist das Gefähl des Lufthungers, das uns veranlasst, die Ausath- 
mung vorzeitig abzubrechen, um wieder „Lult zu schöpfen*. Gerade dadurch aber, dass nun mehr Iuft als gewöhnlich in den Lungen zurückbleibt, muss die folgende Einathmung stärker und der Brustkorb mehr gedehnt werden. Das geht so "staffelförmig" weiter, bis die Brust

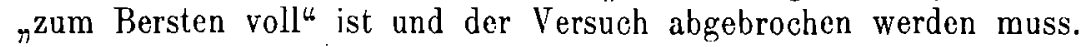

Wenn wir aber den Lufthunger, dem ja weder $\mathrm{CO}_{2}$-Anreicherung noch $\mathrm{O}_{2}$ - Hangel im Blut zu entsprechen brauchen, mit Willen möglichst unterdrückten und uns zwangen, ausgiebig in jeder Respirationsperiode zu exspiriren, so konnten wir dadurch den Eintritt der Lungenblähung ganz wesentlich verzögern.

Wiewohl es feststeht, dass dic Exspirationsmuskeln kräftiger sind als die Inspirationsmuskeln, so wissen wir doch, dass die Exspirationsmuskeln nur die unteren Jungenabschnitte $z u$ verkleinern vermögen. Die von dort verdrängte Luft kann aber in Folge der Stenose der Oeffnungen der oberen Iuftwege nicht genügend entweichen, wird vielmehr in dic oberen Lungenpartien getrieben und dehnt dieselben aus. Dic Lungenblähung ist also wohl stets eine in- und exspiratorische.

Eine Lungendehnung mit exquisit inspiratorischem Charakter haben wir bei den Versuchen mit erschwerter Einathmung und unbehinderter Ausathmung beobachtet. Interessant ist, dass mit Zunahme des unteren Thoraxumfanges der obere abnimmt. Erklärungsversuch: Die starke inspiratorische Dehnung der seitlichen unteren Brustwandpartien führt zu einer erheblichen Juftverdünnung in den entsprechenden Isungenpartien. $\mathrm{Da}$ in Folge der Stenose der Athmungsöffnungen nicht genügend Luft zum Druckausgleich von aussen nachströmen kann, so wird aus den bei dieser erschwerten Inspiration relativ ruhig gestellten Lungenbezirken Lult abgesaugt.

Wir haben also eine oft bis zu $1 / 4$ Stunde währende inspiratorische Ueberdehnung der unteren I,ungenpartien und Verringerung des Umfangs der oberen Thoraxpartien, wobei wir aus Versuchen an uns selbst den Eindruck gewonnen haben, als wäre das Gesammtlungenvolumen ein verringertes. (Ein Bohr'sches Spirometer steht uns leider nicht zur Verfügung.)

Tendeloo nimmt an, dass sowohl der ungeübte Iloboebläser in Folge des hohen Widerstandes im Mundstïck des Instruments, als auch häufig der Kranke während schwerer Hustenanfälle oder Croupparoxysmen zuerst die oberen suprathorakalen Lungentheile durch die energische exspiratorische Verkleinerung der unteren Thoraxpartien aufblähe, dann aber durch die mangelhafte exspiratorische Entleerung der Lungen zu tiefen Athemzügen gezwungen auch noeh die unteren Partien seines Brustkorbes immer mehr erweitere, sodass zu der exspiratorischen Blähung der oberen bald auch eine inspiratorische Ueberdehnung der unteren Lungenabschnitte hinzukomme.

Wir haben versucht dies experimentell mittelst der Stethographie nachzuprüfen. Unsere Versuchsanordnung war so eingerichtet, dass sie etwa dem Hoboeblasen eines Ungeübten entsprach. Ein Receptor sass in der Fossa supraclavicularis, der andere auf dem Sternum. Es gelang uns jedoch nicht zeitliche Unterschiede des Eintritts der Blähung in 
den oberen und dann in den unteren Lungenabschnitten deutlich zu erkennen.

Die häufigste Form der Lungenblähung dürfte bei Stenosen der oberen Luftwege die gemischte sein. Schon in einer früheren Arbeit wiesen wir auf die röntgologischen Untersuchungen Liebermeister's hin, der bei 12 Kindern mit diphtherischer Larynxstenose 11 mal ausgeprägten Zwerchfelltiefstand and geringe Verschiebung der unteren Lungengrenzen fand.

Irgend einen zweckmässigen Reflexvorgang im Sinne Bohr's können wir in diesen Lungonblähungen nicht erkennen. Man kann hier in der Zunahmo des Lungenvolumens keine Anpassung an die Steigerung der respiratorischen Functionen der Lunge sehen, haben doch Morawitz und Siebeck bei ihren Stenoseversuchen Vergrösserung des Lungenvolumens nachgewiesen, ohno gleichzeitiger Aenderung der Blutgase bezw. Zunahme der alveolären $\mathrm{CO}_{2}$-Spannung.

Gehen wir nunmchr über zu der Aenderung des Lungenvolumens bei primärer Veränderung der Blutbeschaffenheit.

Beim Athmen einer $11 \mathrm{pCt}$. $\mathrm{O}_{2}$-haltigen Luft tritt nach Bohr ohne wesentliche Aenderung der Respirationsgrösse und Frequenz (Speck) eine mittlere Füllungszunahme der Lunge bis höchstens 0,4 Liter ein. In die Kategorie dor eigentlichen Lungenblähungen gehört diese Volumzunahme allerdings nicht.

Da trotz dieser $\mathrm{O}_{2}-V^{\prime}$ erdünnung der Einathemluft die $\mathrm{O}_{2}$-Aufnahme ins Blut die gleiche bleibt, so sucht Bohr dies aus der gleichzeitigen Zunahmo des Lungenvolumens zu erklären. Es soll sich dabei um einen Athemreflex handeln, der in der Form der Vergrösserung der Athemoberfläche einen compensatorischen, zweekmässigen Vorgang auslöst. Bohr nimmt einen Reflexvorgang an, der "zwangsmässig dio willkürlich forcirte Ausathmung in einem verhältnissmässig frühen Stadium hemmt". "Zu dieser Hypothese wurde Bohr wohl besonders durch die Vorstellung veranlasst, dass mit der Verminderung der Ausathmungsgrösse die Residualluft entsprechend zunehme. Das stimmt aber nach Hasselbalch's Untersuchungen nicht.

Lässt man die Versuchsperson ein $\mathrm{CO}_{2}$-Gemisch $\left(4,5 \mathrm{pCt} . \mathrm{CO}_{2}\right)$ einathmen, so bekommt sie Lungenblähung. Zugleich mit der Blähung tritt aber eine sehr wesentliche Zunahmo der Athemticfe ein.

Die Entstehung dieser Blähung denkt, sich Bohr ebenso wie beim vorigen Versuch. Bohr geht von dem Gesichtspunkt aus, dass alles, was die Anforderungen an die respiratorischen Functionen der Lunge verstärke, auch deren Mittelcapacität vergrössere. Im speciellen Fall stelle die grössere $\mathrm{CO}_{2}$-Spannung der Alveolenluft, insofern sie die $\mathrm{CO}_{2}$-Ausscheidung aus dem venösen Lungenblut erschwere, grössere Ansprüche an die Arbeit der Lungen. Die Lungenblähung, das ist die Vergrösserung der respiratorischen Oberfläche, compensire diesen Arbeitszuwachs in weiten Grenzen.

Demgegenüber stehen dic Beobachtungen Einthoven's (1892), dass das Einathmen von $\mathrm{CO}_{2}$ Bronchialmuskelcontraction, Athemdruckerhöhung und Lungenblähung verursacht. Diese Resultate werden bestätigt von Beer. 
Also Uebereinstimmung in den Ergebnissen bei Bobr und den eben genannten Forschern, aber grösste Divergenz in der Auffassung über die Entstehung der Blähung. Dort die geistreiche teleologische Hypothese, hier der objective Befund der reflectorischen Bronchiolenverengerung mit consecutiver Blähung durch Vagusreizung.

Ueber Lungenblähung bei Herzkranken hat nun Rubow gearbeitet. Aufbauend auf den Untersuchungsresultaten von Gad, Speck und $\mathrm{Bohr}$, dass $\mathrm{O}_{2}$-Mangel und $\mathrm{CO}_{2}$-Ueberladung des Blutes sowohl ohne wie gleichzeitig mit einer Aenderung der Respirations-Frequenz und -Tiefe Lungenblähung machen, betont er die Bedeutung dieser Blähung bei Herzkranken.

Die Haltung so vieler Herzkranker, aufrecht im Bett sitzend, den Thorax in Inspirationsstellung, dabei ohne hochgradige Vertiefung oder Beschleunigung der Athmung, dieses Bild könne man nur verstehen, wenn man in der Lungenblähung die Circulationserleichterung und die Vergrösserung der respiratorischen Oberfläche sehe. Es entspricht diese Auffassung den bekannten Bohr'schen Gedankengängen. Bohr nimmt an, dass die Lungenblähung zweckmässig sei, weil durch sie die respiratorische Oberfläche vergrössert werde. Dies sei für den respiratorischen Stoffwechsel von wesentlicher Bedeutung, indem nun die Lungenzellen unter günstigeren Bedingungen wirken. Ferner aber würden durch die Zunahme der Mittelcapacität die Capillargefässe erweitert und dadurch die Circulation des Blutes durch die Lungen erleichtert. Dadurch vermindere sich der Widerstand im kleinen Kreislauf und damit die Arbeit des rechten Herzens.

Dementsprechend sieht Rubow in der Zunahme des Lungenvolumens eine Erleichterung der 'I'hätigkeit des angestrengten rechten Ventrikels bei decompensirten Mitralfehlern, indem durch die Entfaltung der Alveolen die Capillaren gestreckt und dadurch die Blutströmung nach dem linken Herzen erleichtert werde.

Sowohl bei Neurose der Herznerven als bei organischen Herz- und Gefässveränderungen treten nicht selten plötzliche schwere Anfälle von Athemnoth (Asthma cardiale) auf. Die dabei krampfhaft eingehaltene Inspirationsstellung des Thorax mit gleichzeitiger Lungenerweiterung schafft nach Rubow dem Kranken dadurch Erleichterung, dass die vom versagenden linken Ventrikel nicht weitergetriebene Blutmenge nun eher in den Lungen Platz findet.

Diese Zweckmässigkeitsvorstellungen unterwarf Tendeloo (1908) an der Hand von Experimenten und Berechnungen einer strengen Kritik.

Wir wissen nicht, ob im Allgemeinen Vergrösserung des Lungenvolumens auch Zunahme der Capacität der Lungencapillaren bedeutet. Und dann vergrössern sich doch auch bei Eintritt einer Lungenblähung durchaus nicht alle Lungenalveolen gleichmässig. Ebensowenig ist es erwiesen, dass die bei Lungenblähung auftretende Streckung der Capillargefässe die Stromgeschwindigkeit erhöht. Und wenn die Stromgeschwindigkeit wirklich zunehmen würde, so kennt man wieder die Zeit nicht, welche das Hämoglobin unbedingt nöthig hat, um sich mit $\mathrm{O}_{2}$ sättigen zu können. 
Man wird also nur sagen können: Eine mässige Volumzunahme der Lunge, wie wir sie beim Athmen $\mathrm{O}_{2}$-armer bezw. $\mathrm{CO}_{2}$-reicher Luft, ferner bei Herzkranken mit dauernder Cyanose und leichter Athemvertiefung sehen, kann die Blutcirculation in bestimmten Lungenbezirken erleichtern.

Es ist jedoch vielfach klinisch und pathologisch-anatomisch bewiesen, dass eine stärkere Lungendehnung, wie sie z. B. bei Stenosen der oberen Luftwege (Liebermeister), Asthma bronchiale und Asthma cardiale beobachtet wird, die Function der befallenen Lungentheile in jeder Hinsicht empfindlich schädigt. Die Blassheit, d. h. absolute Blutarmuth acut gedehnten Lungengewebes ist bekannt, ebenso die Herzdilatationen, die so häufig im Asthmaanfall auftreten.

Als Ursachen der an sich mässigen Füllungszunahme der Lungen schwer Herzkranker kommen verschiedene Punkte in Betracht. Das Respirationscentrum bekommt durch eine zu langsame Circulation zu wenig aber im übrigen normal arterialisirtes Blut (Rubow). Ferner kann die Blutstaung in der Lunge den Luftraum in den Bronchiolen weitgehend reduciren und dadurch die $\mathrm{CO}_{2}$-Ausscheidung und $\mathrm{O}_{2}$-Zufuhr beeinträchtigen. So kommt es zur Vertiefung der Athmung und zum Gefühl der Athemnotb. Vermuthlich sind Lufthunger, Angst und innere Unruhe auch ein Anlass dazu, dass die Kranken den Thorax in Inspirationsstellung halten. Durch diese Anstrengung bekommen sie dann wieder das Gefühl der erschwerten Athmung, "Athemnoth" und athmen entsprechend tiefer ein. Und noch eins: Bei inspiratorisch erweiterten Lungen ist die Luftzu- und Abfuhr aus den durch Stauungshyperämio verengten Alveolen und Bronchien erleichtert. Dies könnte ebenfalls ein Grund der instinctiv unter Anstrengung eingehaltenen Inspirationsstellung sein.

Das Hypothetische der Annahme Bohr's, die Zunahme des Lungenvolumens entstehe in Folge der gesteigerten Function der Lunge, ergaben schon die spirometrischen Untersuchungen von Bohr's Schüler Hasselbalch über den Reiz, den die plötzliche Aenderung der umgebenden Luft- bezw. Wassertemperatur auf das Lungenvolumen ausübt. Hassclbaleb constatirte nämlich, dass das Ablegen sämmtlicher Kleider bei $14^{\circ} \mathrm{C}$. Zimmertemperatur eine Zunahme des Lungenvolumens hervorruft, obwohl weder die respiratorische Arbeit der Lunge (respiratorischer Stoffwechselversuch!) noch die $\mathrm{CO}_{2}$-Spannung in der Alveolenluft zunimmt.

Nach den bedeutungsvollen Experimenten Einthoven's und Beer's steht es fest, dass periphere und reflectorische Vagusreizung Contraction der gesammten Bronchialmuskulatur und dadurch unter Athemdruckerhöhung Lungenblähung hervorrufen. Es könnte also die unter der Wirkung sensibler Reize und psychischer Einflüsse einsetzende Lungenblähung durch eine reflectorische Erregung des Vagus erklärt werden. Dafür spricht besonders der Umstand, dass jone Lungenblähung sofort verschwindet, sobald die Vagi durchtrennt werden.

In der klinischen Pathologie beobachten wir die Wirkung solcher plötzlich einsetzenden Verengerung des Bronchiallumens beim Asthma bronchiale. 
Hier werden die durch Bronchialmuskelkrampf (Secretionssteigerung und vasomotorische Gefässerweiterung) verengten Bronchiolen nur durch kräftige Thoraxdehnung genügend erweitert, während der Ausathmung aber noch mehr verengert. Bei forcirter Inspiration sind die Bronchiolen für die Luft hinreichend durchgängig, während die exspiratorische Verstärkung des Intrapulmonaldruckes durch die Steigerung der Verengerung der Bronchiallumina eine wesentliche Erschwerung für den respiratorischen Luftstrom bedeutet. Die Luft verlässt nur langsam die Lungen, die Exspirationsdauer ist erheblich verlängert. Aber ehe die eingeathmete Luft wieder ganz entfernt ist, setzt die neue Inspiration ein. So wird mit jeder Respirationsperiode ein Quantum Luft in der Lunge zurückgehalten und der Thorax geräth in die bekannte Inspirationsstellung.

Die Lungenblähung ist hier eine vorzugsweise inspiratorische (Tendeloo). Davon konnte ich mich zur Zeit der Niederschrift dieser Arbeit selbst an Kranken mit Bronchialasthma überzeugen. Die Einathmung sei sehr erschwert, die Ausathmung gehe leicht, versicherten mich die Kranken. Schon die ganze Körperhaltung weist darauf hin; sie dient der Erleichterung der Inspiration. Die Ausathmung stellt eine Combination dar von Entspannung der Einathemmuskeln und vorsichtiger musculärer Exspiration. Die Unzweckmässigkeit einer raschen forcirten Exspiration bezw. die dadurch hervorgerufene Compression der Bronchiolen konnten wir an unseren Kranken mit Leichtigkeit demonstriren. Zwar gelang es ihnen zu Beginn solcher Exspiration durch energische Contraction der Bauchmusculatur ein geringes Quantum Luft zu exspiriren. Dann aber trat ein plötzlicher Stillstand in der Exspirationsbewegung ein, der Kranke war völlig unfähig, noch weiterhin Luft aus den Lungen auszupressen. Tendel oo vermuthet mit Recht, dass bei einer derartigen allgemeinen Bronchiolenverengerung die abnorme Brustkorbdehnung und damit die Lungenblähung bis zu einem gewissen Grade einen compensatorischen Vorgang darstelle, denn es scheint, als $o b$ erst dann, wenn die Iungen und damit auch die verengerten Bronchiolen statisch einigermaassen gedehnt sind, durch die heftigen Einathembewegungen wirklich genügend Luft die Bronchiolen passiren könnte. Und auch die Abgabe der Luft aus den Lungen kann natürlich nur aus diesem Dehnungszustand Gewinn ziehen.

Ausgelöst wird also der einzelne Asthmaanfall durch einen Reiz auf den Vagus, der von den verschiedensten Stellen des Körpers aus erfolgen kann (A. uterinum, uraemicum etc.). Diese Vagusreizung erzeugt nun den Bronchialmuskelkrampf und, wie Talma und Zwaardemaker nachweisen konnten, auch Contractionen der Larynx- und Trachealmusculatur.

Ist der Bronchialmuskelkrampf heftig und die Exspiration dadurch besonders ungenügend, so kann es durch die starke Retention der $\mathrm{CO}_{2}$ im Blut zu einem Circulus vitiosus kommen, insofern die $\mathrm{CO}_{2}$ nun ihrerseits das Vaguscentrum in der Medulla reizt und dadurch den Bronchialkrampf nur noch vermehrt.

Mit dem Asthma cardiale hat sich auch F. A. Hoffmann eingehend beschäftigt. 
Ausser durch primär mechanische Circulationsstörungen und deren Folgen können nach Hoffmann derartige Paroxysmen einer cardialen Dyspnoe auch eintreten durch "Schädigung des nervösen Apparates im Herzen, welcher einen directen Einfluss auf das Respirationscentrum ausübt". Diese Schädigung wird z. B. hervorgerufen durch Blutdruckerhöhung bei Arteriosklerose, ferner durch eine Reihe von toxischen Stoffen (alimentäre: Asthma dyspepticum, renale: Asthma uraemicum. Blei, Chlor: Blei-Chlorasthma).

Die Veranlassung zum Ausbruch eines cardio-asthmatischen Anfalls liegt also nach diesem Autor theils in mechanischen, theils in reflectorischen Vorgängen. Hoffmann schildert zwar bei der Beschreibung des Anfalls wie der Kranke dasitzt, „mühsam nach Athem ringend, die Arme aufgestützt, mit aufwärts gezogenen Schultern, um die Muskeln des Schultergürtels für die Erweiterung des oberen Thorax zur Verfügung zu haben". Auch bestätigt der Autor die Erweiterung der Lungengrenzen während des Anfalls, aber bei seinen Ueberlegungen über die Ursachen der paroxysmalen Dyspnoe berücksichtigt er diese ganz specifische Form der Athemnoth gar nicht und daher haben seine Ueberlegungen über Irradiation von Erregungen vom Herz her auf das Respirationscentrum etc. nur bedingtes Interesse.

Nach der heutigen Ansicht kann die asthmatische Lungenblähung nur durch eine ziemlich hochgradige Bronchiolenverengerung erklärt werden und daher muss deren Entstehung und Wesen in erster Linie klar gelegt werden.

Eine mechanische, d.h. durch Blutstaung und erhöhten Blutdruck bedingte Bronchiolenverengerung ist nach Einthoven's überzeugenden Experimenten als directe Ursache der Lungenb]ähung ausgeschlossen. Die reflectorische Verengerung durch Contraction der Bronchialmuskeln jst nur auf Vagusbahnen möglich.

Es ist daher die Auffassung F.A. Hoffmann's, dass der Vagus mit der paroxysmalen Dyspnoe nichts zu thun habe, nur insoweit gerechtfertigt, als man eine doppelseitige Vaguscompression durch Tumoren bezw. Vaguslähmung nicht verantwortlich machen kann für umschriebene Anfälle von Lungenblähung mit Tachycardie etc., die sich im Lauf von Jahren und Jahrzehnten nur gelegentlich wiederholen.

Solche Anfälle haben Tuczek, Kredel, Honigmann, A. Hoffmann und Langer beschrieben.

Tuczek, Kredel und Langer nahmen als Ursache dieser Erscheinung eine periphere Reizung der Lungen- und gleichzeitige Lähmung des Herzfasern des Vagus an. Die Reizung des Vagus erfolge in Form einer Compression des Nerven durch geschwollene Bronchialdrüsen. Da nun aber die Lungenblähung und Tachycardie nur in Anfällen auftreten, so müssten dementsprechend die Bronchialdrüsen Jahre lang von Zeit zu Zeit an- und abschwellen, um die vorübergehende Compression, d. h. Anfälle auslösen zu können. Darin liegt das Missliche der Annahme solcher peripheren Vagusreizung.

Wir sind vielmehr mit Aug. Hoffmann der Ueberzeugung, dass ebenso wie die den Anfall begleitende Tachycardie und die vaso- 
motorischen Symptome (Schweissausbruch, Hautröthung), sowie dyspeptische Erscheinungen (Erbrechen, $\Lambda$ ufstossen) so auch dic Lungenblähung in diesen Fällen ihren Aus]ösungsort in der Medulla oblongata hatte.

Wir bezwoifeln daher auch, dass das echte Asthma dyspepticum stets unter das Asthma cardiale zu subsummiren ist, wie F. A. Hoffmann will.

Bei diesen Asthmaanfällen soll nämlich nach F. A. Hoffmann eine reflectorische Erregung vom Magen und Darm aus zuerst das Gefässnervencentrum treffen, dabei komme es zur Blutdrucksteigerung. Diese wiederum führe zu einer Mitbetheiligung des Herzens und vom Herz aus soll die Erregung auf das Respirationscentrum irradiiren. So komme die paroxysmale Dyspnoe zu Stande. Irradiationen vom vasomotorischen Centrum direct auf das Respirationscentrum lehnt Hoffmann ab.

Die Hauptsymptome beim Asthma dyspepticum: hochgradige Jungenblähung, Angstgefühle, Kälte der Extremitäten, Schweissausbrüche, Herzklopfen sind vielmehr der Ausdruck einer mehr weniger gleichzeitigen Erregung des Vasomotoren-, Herzbewegungs- und Vaguscentrums in der Medulla oblongata. Die Ursache liegt nach allgemeiner Annahme in einer Autointoxication, die hervorgerufen ist durch Verdauungsschädigungen.

Zuelzer, der die obengenannten klinischen Symptome in mehreren Fällen beobachtet und als Vagusneurose bezeichnet hat, betont als Ursache vorwiegend hartnäckige Verstopfung. In einem seiner Fälle wich die Lungenblähung 15 Minuten nach einer Atropineinspritzung, also wohl durch Lösung eines Bronchialmuskelkrampfes (Einthoven, Beer, Brodie).

Hierher gehören auch die Lungenblähungen bei Angstzuständen Geisteskranker, wie sie schon vor 30 Jahren Griesinger und neuerdings Ziertmann beschreibt. Die Symptome: Iungenblähung, Blutdruckerhöhung, Blässe und Kälte der Haut, Schweiss, weite Pupillen führt Ziertmann mit Recht auf eine Erregung des Vasomotoren- und Vaguscentrums zurück, was ja auch der Auffassung Lähr's entspricht, dass ,der Mechanismus der Angst auf Reizung von Oblongatacentren" zurückzuführen sei.

Den Fntwicklungsgang aller dieser reflectorischen Lungenblähungen verstehen wir an Hand der Ergebnisse unserer Stenoseversuche. Einerlei ob eine normale oder eine Uebererregbarkeit, Neurose der Lungennerven vorliegt, so resultirt wohl meist aus einer Reizung des Vaguscentrums durch Giftstoffe, psychische Einflüsse oder durch wesentlichen $\mathrm{O}_{2}$-Mangel und $\mathrm{CO}_{2}$-Anreicherung des Blutes oine reflectorisehe Verengerung der Luftwege. Jedenfalls tritt immer die Jungenblähung ein durch inspiratorische Dehnung der Lungen ohne entsprechende exspiratorische Verkleinerung. Es kommt zu Vertiefung der Inspirationen, weil nul so die Luft dureh die verengten Wege durchgesogen werden kann. Nun aber spielt auch hier die bei den einzelnen Kranken verschieden starke Empfindung und ebenso differente Reaction auf das Gefühl der Athemcrschwerung eine wichtige Rolle. Abgesehen von Bronchialasthmatikern, die im Laufe der Jahre gelernt haben, im Anfall die Athmung entsprechend zu reguliren (siche unsere Stenoseversuche) geben die Versuchs- 
personen bezw. Kranken, die von einer plötzlichen Verengerung der Juftzuführenden Wege befallen werden, dem Luftbedürfniss schrankenlos nach. Sie inspiriren so tief wie möglich und lassen sich nicht die Zeit zu genügender Ausathmung. So entsteht Lungenblähung, die ihrerseits wieder die Athemnoth vermehrt. Damit schliesst der Circulus vitiosus, wie wir ihn oben schon besprochen haben.

Im Gegensatz zu der Sungenblähung bei Verengerung der Trachea oder der Athemöffnungen kommt es hier zu keiner Erweiterung der oberen Lungenpartien durch Verstärkung der Exspiration. Hier führt, wie wir an dem Beispiel unserer Bronchialasthmatiker zeigen konnten, der Versuch einer forcirten Exspiration durch Steigerung des Intrapulmonaldrucks zum Verschluss der verengten Bronchiolen und Stillstand der Ausathmungsbewegung.

Bei dem ron Jamin beobachteten Hysteriker lag ein schwerer Erregungszustand der Respirationscentren vor. (Ob reflectorische Bronchialverenrerung bestand, ist nicht zu entscheiden. Am ehesten wird man an eine inspiratorische Lungenblähung wie bei jeder anderen intensiven Körperanstrengunğ denken dürfen.

Eine Erklärung der durch Körperanstrengung bedingten Volumzunahme der Junge haben wir noeh nicht gegeben. The wir dazu übergchen, möge noch einmal darauf hingewiesen sein, dass es sich bei diesen Yolumzunahmen nach den bisherigen spirometrischen Untersuchungen nur um höchstens $100 \ldots 500$ ccm luft handelt, währeud \%. B. schon der Luftwechsel bei völig ruhiger Athmung $1 / 2$ liter beträgt.

Die Bohr'sche Aufassung dieser Erhöhung der Mitteleapacitat ist uns schon bekannt. lis ist auch hier der ,zwangsmässigr dio willkürlich forcirte Exspiration hemmende Reflex", weleher die Volunzunahme za Stande bommen lässt, dadureh die Capillaren erweitert und so einer Ueberanstrengung des Herzens vorbeugt. Gegen diese Theorien spricht schon, dass nach Bohr's und II asselbaleh's Protokollon häufig nach der Anstrengung das Volumen der Linnge schon lange wieder zur Norm zuriickgekehrt ist, während Frequonz und Tiefe der Athemzïge ebenso wie die Pulsfrequenz noch um das Mehrfache gesteigert sind.

Durch die Iuskelarbeit wird die CO.-Spannung in den Alveolen und im Arterienblut erhöht. Dadurch wird das Respirationscentrum errogrt. Die kolge davon ist, dass die Athemzäge solange verstärkt werden, bis die Spamung der $\mathrm{CO}_{2}$ in Blut und Alveolarluft wieder ihren früheren Werth erreicht hat (Haldane, Priestley). Nach Zuntz und Geppert rufen die bei der Muskelarbeit entstehenden Säuren die Verstärkung der Athmung hervor. Wie dom auch sein mag, durch die tieferen Inspirationen werden der elastische Brustkorb und die Jungen stärker gedehnt und hätten eine längere Zeit für die Verkleinerung zum normal-exspiratorischen Volumen nöthig. Diese Zeit wird ihoen aber durch die Athemfrequenzzunabme nicht gegönnt. Die neue Einathmung erfolgt, ehe die inspiratorisch gedehnten Alveolen sich exspiratorisch wieder röllig zusammengezogen haben, also ehe die elastische Nachwirkung zu einer vollkommenen geworden ist. 
Dieser Vorgang wiederholt sich und so bleibt Luft im Thorax zurück. Natürlich kehren auch bei Schluss der Anstrengung Lunge und Thorax nicht sofort, sondern erst allmählich zu ihrem normal-exspiratorischen Volumen zurück; die unvollkommene elastische Nachwirkung wird ganz allmählich zur vollkommenen.

Wir haben es also hier bei Fehlen jeder Exspirationserschwerung mit einer rein inspiratorischen Volumvergrösserung der Lunge zu thun.

Wir haben uns nun zu fragen, was wird aus Thorax und Lunge, die durch häufige Körperanstrengungen oftmals in einen Dehnungszustand gebracht worden waren? Tritt hier mit der Zeit eine Ueberdehnung der elastischen Gewebe auf? Wir wissen, dass Thorax- und Lungeninhalt von Sportsleuten mit fortschreitender Körperübung zunimmt. Nun stellt Tendeloo den Satz auf, dass es mit den elastischen Lungenelementen gehe, wie mit einer Gummimembran, deren elastische Nachwirkung durch sehr häufige Dehnungen allmählich unvollkommen wird, was wir dann an der Zunahme ihrer Länge sehen. Dagegen ist einzuwenden, dass bei Sportsleuten, bei denen es sich in erster Linie um eine inspiratorische Ueberdehnung der caudalen lateralen und sterno-parasternalen Lungentheile handeln müsste, echtes Emphysem pathologisch-anatomisch bisher nicht beobachtet worden ist. Die Zunahme des mittleren Lungenvolumens bei ihnen als Ausdruck vielfacher intensiver Lungenthätigkeit wird also wohl daher kommen, dass sich das Plus an Luft ganz gleichmässig auf die Lungenalveolen vertheilt, und dass eben bei dieser Zunahme des Thoraxumfanges mehr Alveolen als früher ständig einen gewissen Luftgehalt auch in Körperruhe behalten. Auch die ausserordentlich häufige exspiratorische Dehnung der Alveolen der oberen Lungenabschnitte, wie wir sie bei Trompetenbläsern sehen, führt nach den Untersuchungen von Pretin und Leibkind, sowie von Fischer keineswegs zu chronischem Lungenemphysem. Echtes Emphysem stelit sich nach den Untersuchungen dieser Autoren, sowie ganz besonders nach den Anschauungen, die F. A. Hoffmann in seinem ausgezeichneten Werk über Lungenemphysem niedergelegt hat, nur dann ein, wenn bei diesen Leuten Jahre lang anhaltende Entzündung mit secundärer Verengerung der Bronchien und häufigem anhaltenden Husten besteht. Auch wir glauben, dass das chronische, rareficierende Emphysem nur in der Minderzahl der Fälle durch temporäre Ueberdehnungen bestimmter Lungenabschnitte eintritt. Haben doch Sudsuki und Köhler durch künstliche Trachealstenose beim Thier zwar starke Lungenerweiterung und bemerkenswerther Weise auch Hypertrophie des rechten Herzens hervorgerufen, aber dabei weder Obliteration von Alveolarsepten noch irgendwelche Schädigung der elastischen Elemente gefunden.

Die schwere, inspiratorische Blähung der gesunden Lunge, die auftritt bei Verstopfung des Hauptbronchus der anderen Lunge, entsteht allerdings ohne Verengerung der Luftwege der gesunden Lunge, aber hier handelt es sich um ein vicarirendes Emphysem, das der Schrumpfung der verschlossenen Lunge entsprechend langsam zunimmt.

Fassen wir die wichtigsten Punkte noch einmal zusammen, so wäre zu sagen: Ob man den Thorax erst bewusst in Inspirationsstellung bringt 
und nun eine Zeit lang ruhig aus und ein athmet oder ob man bei Uebergang von normaler zu vertiefter Athmung absichtlich bezw. aus $\mathrm{Cn}$ geschicklichkeit die Ausathmung unzureichend ausführt und so die Lungen mit Juft vollpumpt, ist im Lffect gleichgültig: Mit der Rückkehr zur normalen, ruhigen Athmung verschwindet eine derartige Veränderung des Lungenvolumens sofort.

Eine mässige Volumzunahme der Lunge bis höchstens $500 \mathrm{ccm}$ beobachten wir bei und nach Körperanstrengung. Is handelt sich dabei um einen rein inspiratorisch entstandenen Leberdehnungszustand hauptsächlich der unteren seitlichen 'Thorax- und Lungenpartien.

Die Verkleinerung dieser Partien bis zum normal exspiratorischen Volumen erfolgt meist innerhalb der 1. Viertelstunde nach Beendigung der Körperanstrengung.

Weder bei jencr willkürlichen Aenderung des Jungenrolumens noch bei derjengen dureh cinmalige Körperanstrengung ist eine bleibende, inspiratorische Leberdehnung der betreffenden Brustwand- und Lungenabschnite bisher beobachtet worden. Ebenso wenig ist darüber etwas bekannt, dass häufig wiederholte Körperanstrengungen die Brustwandund Lungenelasticitä schädigen.

Sehwere Lungenblähung tritt, normale liungen vorausgesetzt, nur ein bei rellectoriseher oder mechaniseher (Bronchiolitis) Verengerung des Bronehialsystems sowie bei stenosen der oberen luftwege bezw. deren Oeffinumgen.

Handelt es sich um eine allgeneine Verengerung des Systems derkleineren Bronchien, so trägt die lungenblähung rein inspiratorisohen Charakter.

Bei den hochsitzonden stenosen der oberen Juftwege und deren Oeffnungen greht meist die inspiratorische Dehnung der lateralen, eaudalen Thoraxpartien mit einer axspiratorischen Blähung der oberen Lungenabsehnitte einher. Voraussetzung ist, dass die Aus- und Einathmung gleichmässig stark oder nur die Ausathmung ersehwert ist.

Nur bei diesen sehweren Lungenblähungen kaun es sehon nach einmaliger licherdehnung zu dauerndem Elasticitätsverlust der betreffenden Partien kommen, d. h. zu cinem echten Jungenemphysem (Keuchbusten, kirinken).

Der Grad der Lungenblähnug and die Sohnelligkeit ihres Eintritts hängen von der Siarke der Inspirationen ab und diese wieder entsprechen den objectiven Veränderungen, dabei aber aush dem bei den einzelnen Versuchspersonen und Kranken - ceteris paribus -- so sehr verschiedenen Luftbedürfniss bezw. der Empfindlichkeit gegen Athemerschwerung.

\section{li i 1 o r a $\mathbf{t}$ II r.}

Albu und Caspari, Deutsche med. Wochenschr. 1903. No. 3. S. 259.

Becki und bohan, Jeber Veränderung der llerzarösse in heissen und kalten Bade. Münrhn. med. Wochenchr. 1909. No. 4.

Beer, Ueber den Finfuss der peripheren Vagusreizung auf die Lunge. Du Bois Reymond's Archiv. 1892. 
Bohr, Wien. med, Wochenschr. 1907.

Derselbe, Die functionelle Bedeutung des Lungenvolumens in normalen und pathologischen Zuständen. Aus d. Verhandl. d. Gesellsch. Mü̈nchn. Naturf. ฉ. Aerzte. 1907. 1 u. 2. IX. C.

Derselbe, Die functionellen Aenderungen in der Mittellage und Vitalcapacität der lungen. Deutsches $\Lambda \mathrm{rch}$. f. klin. Med. 1907. Bd. 88.

Derselbe, Zur Theorie der Entstehung des Lungenemphysems. Centralbl. f. d. ges. Physiol. u. Pathol. des Stoffwechsels. X. F. 1908. 3. No. 7.

Einthoven, Leber die Wirkung der Bronchialmuskeln, nach einer nouen Methode untersucht, und über $\Lambda$ sthma nervosum. Pflüger's Arch. f. d. ges. Physiologie. 1892. Bd. 51.

Gad, Respiration. Eulenburg's Realencyklopädic. Bd. 20. S. 367.

Hasselbaich, 1. Leber die Einwirkung der Temperatur auf dio vitaie Mittellage der Lungen. - 2. Ueber die Totalcapacität der Iungen. Deutscbes Arch. f. klin. Med. Bd. 93. H. 1 ท. 2.

Hofbauer und Holzknecht, Zur Physiologie und Pathologie der $\Lambda$ thmung. Mittheil. a. d. Laborat. f. radiolog. Diagnostik u. Ther. im k. k. allgem. Krankenhaus in Wien. Gust. Fischer, Jena. 1907.

H ofbauer, Zur Frage nach der Entstehung der Lungenblähung. Deutsche med. Wochenschr. 1908. No. 51.

Hoffmann, F. A., Emphysem und Atelectase. Spoc. Path. u. Therapie. Nothnagel. 1900. Bd. XIV. II. 3. Wien.

Derselbe, Asthma cardiale. Deutsche Klinik. 1907. Bd. IV.

Hoffmann, Aug., Die paroxysmale Tachycardie. Wiesbaden. 1900. Bergmann.

Jamin, Zwerchfell und Athmung. Atlas und Grundriss der Röntgendiagnostik in der inn. Med. Dr. Groedel, München. Lehmann's Verlag. 1909.

Köhler, Leber die Compensation mechanischer Respirationsstörungen und die physiologische Bedeutung der Dyspnoe. Archiv f. exp. Pathol. u. Pharm. Bd. 7. S. 1. Kredel, Zur Lehre ron den Vagusneurosen, Deutsches Arch. f. klin. Med. Bd. 30. Lähr, Die Angst. Berl. Klinik. 1893. H. 58.

Liebermeister, Zur normalen und pathologischen Physiologie der Athmungsorgane. Deutsch. med. Wochenschr. 1908. S. 1669.

Morawitz und Siebeck, Die Dyspnoe durch Stenose der Luftwege. I. Gasanalytische Untersuchungen. Deutsches $\Lambda$ rchiv f. klin. Med. Bd. 97. II. 3 u. 4.

Moritz, Ceber functionelle Verkleinerung des Herzens. Münchn. med. Wochenschr. 1908. No. 14.

Derselbe, Ueber orthodiagrapllische Untersuchungen am Hermen. Ebendas. 1902. H. 1. No. 1.

Derselbe, Ebendas. 1901.

Moritz und Dietlen, Ceber das Verhalten des Herzens nach langdauerndem und anstrengendem Radfahren. Fibendas. 1908. No. 10.

Pretin und Leibkind, Kann durch Glasblasen ein Lungenemphysem erzeugt werden? Ebendas. 1904. H. 13.

Rubow, Untersuchungen über die Athmung bei Herzkrankheiten. Ein Beitrag zum Studium der Pathologie des kleinen Kreislaufs. Deutsebes Arch. f. klin. Med. 1908 . H. 3 u. 4.

Sänger, Ueber Asthma und dossen Behandlung. Berlin 1910. Karger.

Derselbe, Deutscho Aerzte-Zeitung. 1906.

Schieffer, Ueber Herpvergrösserung in Folge Radfahrens. Deutsches Arch. f. kliu. Med. Bd. 89. H. 5 u. 6. 1907. 
Nachweis, Entstehung und Bedeutung der temporären Lungenblähung. 517

Scbott, Zur Frage der acuten Herzüberanstrengung. Münch. med. Wochenschr. 1908. No. 18.

Derselbe, Ebendas. 1890.

Siebeck, Die Dyspnoe durch Stenose der Luftwege. II. Die Einstellung der Mittellage der Lunge. Deutsches Archiv f. klin. Med. Bd. 97. H. 3 u. 4.

Speck, Physiologie des menschlichen Athmens. Leipzig 1892.

Tendeloo, Die functionelle Bedeutung des Lungenvolumens in normalen und pathoIogischen Zuständen. Aus d. Verhandl. deutsch. Naturf. u. Aerzte. 1907. 1. 2. iX. C. $74 \mathrm{~b}$.

Derselbe, Studien über die Ursachen der Lungenkrankheiten. Wiesbaden. J. Bergmann. 1902.

Tuczek, Ueber Vaguslähmungen. Deutsches Arch. f. klin. Med. Bd. 21.

Ziertmann, Ueber acuteLungenblähung bei Angstzuständen Geisteskranker. Münchn. med. Wochensehr. 1894. No. 38 u. 39.

Zuelzer, Ueber Vagusneurose. Centralbl. f. d. ges. Physiol. u. Pathol. d. Stoffwechsels. N. F. 1908. 3. No. 3. 